\title{
A Small-World Optimization Algorithm Based and ABC Supported QoS Unicast Routing Scheme*
}

\author{
Xingwei Wang, Shuxiang Cai, and Min Huang \\ College of Information Science and Engineering, Northeastern University, Shenyang, \\ 110004, P.R. China \\ wangxw@mail.neu.edu.cn
}

\begin{abstract}
In this paper, by introducing knowledge of fuzzy mathematics, probability theory and gaming theory, a QoS unicast routing scheme with $\mathrm{ABC}$ supported is proposed based on small-world optimization algorithm. Under inaccurate network status information and imprecise user QoS requirement, the proposed scheme uses the range to describe the user QoS requirement and the edge parameter, introduces the user satisfaction degree function, the edge evaluation function and the path evaluation function, trying to find a QoS unicast path with Pareto optimum under Nash equilibrium on both the network provider utility and the user utility achieved or approached. Simulation results have shown that it is both feasible and effective with better performance.
\end{abstract}

Keywords: NGI(Next Generation Internet), QoS(Quality of Service), Unicast routing, ABC (Always Best Connected), Small-world optimization algorithm, Nash equilibrium, Pareto optimum.

\section{Introduction}

Recently, with the growth and convergence of Internet, multimedia content and mobile communication technology, NGI (Next Generation Internet) is becoming an integrated network [1-4] converged seamlessly by heterogeneous multi-segment multi-provider sub-networks, such as terrestrial-based, space-based, fixed and mobile sub-networks, etc. Its backbone and access links become diversified. Several kinds of links may coexist on each hop for the user to choose along the end-to-end path. It is possible for the user to be ABC (Always Best Connected) [3-4] to NGI in the course of communication, that is, the user can connect with NGI anytime, anywhere in the currently best way and can switch to the better way adaptively and transparently whenever it comes forth, and thus the so-called global QoS (Quality of Service) roaming should be supported seamlessly [5].

In NGI, QoS routing is essential and $\mathrm{ABC}$ should be supported [4]. However, some characteristics of NGI, such as its heterogeneity and dynamics, influence of terminal and even network mobility, unavoidable message transfer delay and its uncertainty,

\footnotetext{
This work is supported by the National High-Tech Research and Development Plan of China under Grant No. 2006AA01Z214; the National Natural Science Foundation of China under Grant No. 60673159; Program for New Century Excellent Talents in University; Specialized Research Fund for the Doctoral Program of Higher Education; the Natural Science Foundation of Liaoning Province under Grant No. 20062022.
} 
etc., make it hard to describe the network status used when routing in NGI exactly and completely. On the other hand, the user QoS requirements are affected largely by a lot of subjective factors and often can not be expressed accurately, therefore the flexible QoS description should be provided. ABC means a user can get the best available connection anytime, anywhere, however, 'best' itself is a fuzzy concept, depending on many factors, such as user QoS requirement, cost a user willing to pay, user preference, terminal ability and access network availability, etc. In addition, with the gradual commercialization of network operation, $\mathrm{ABC}$ is not a user's own wishful thinking and thus need to consider both the network provider profit and the user profit with both-win supported [6].

It has been proved that the problem of finding a path subject to constraints on two or more additive or multiplicative metrics in any possible combination is NPcomplete [7]. There are already many heuristic and intelligent optimization algorithms used to solve this problem. In [8], the edges that do not meet with the bandwidth constraint are cut off from the graph at first, and then Dijkstra algorithm [9] is used to find the bandwidth-constrained least-delay path with delay as weight. In [10], a distributed heuristic algorithm is proposed, which constructs a delay vector and a cost vector for each node to help find the path. In [11], a comprehensive parameter that is the probabilistic combination of cost and delay is introduced and used when routing. In [12], QoS parameters are defined as path-correlative non-negative variables and are divided into two classes: QoS sensitive and non-sensitive, so that the routing computation can be simplified. In [13], a route pre-computation scheme is proposed based on Bellman-Ford algorithm [14], and the substantial improvement in terms of computational complexity has been achieved by quantizing the cost function. In [15] and [16], a distributed delay-constrained algorithm and a multi-constrained source routing algorithm are proposed respectively. In [17], several pseudo-polynomial time algorithms have been proposed to solve the delay-constrained least-cost routing problem. On the other hand, many optimization algorithms, such as genetic algorithm, ant colony algorithm, and simulated annealing algorithm, have been used to solve QoS routing problem in order to find the optimal or near-optimal QoS route [18-20].

However, the above proposed schemes do not consider sufficiently realizing a QoS unicast routing mechanism with both-win of the network provider and the user supported under imprecise network status and inaccurate user QoS requirement from $\mathrm{ABC}$ viewpoint. In this paper, by introducing knowledge of fuzzy mathematics, probability theory and gaming theory, a QoS unicast routing scheme with ABC supported is proposed. In order to deal with imprecise network status information and flexible user QoS requirement, it uses range to describe the user QoS requirement and the edge parameter and introduces the user satisfaction degree function, the edge evaluation function and the path evaluation function. Based on small-world optimization algorithm, it tries to find a QoS unicast path with Pareto optimum under Nash equilibrium on both the network provider utility and the user utility achieved or approached. Simulation results have shown that the proposed scheme is both feasible and effective with better performance.

\section{Problem Description}

In this paper, the adopted network model, routing request, edge parameter probability model, user satisfaction degree function, edge and path evaluation function, gaming 
analysis and mathematical model are the same as those in [21]. Due to limited space, please refer to [21] for their detailed descriptions.

\section{Algorithm Design}

Small-world optimization algorithm [22] optimizes its solution searching process based on small world phenomenon, regarding optimization process as information transfer from a candidate solution to the optimized solution in searching space. The proposed QoS unicast routing scheme based on small-world optimization algorithm is described as follows.

\subsection{Basic Definition}

In Small-world optimization algorithm, call a candidate solution as a transfer node. In this paper, $s$ represents a transfer node and $S$ represents a transfer node set, $s \in S$. $s=s_{1} s_{2} \cdots s_{|E|}$ adopts binary encoding, if one bit is 0 , its correspond edge is in $s$, otherwise not in $s$. A node is generated randomly, that is, each bit of its codes is set to be 0 or 1 randomly.

The fitness function of $s$ is defined as follows:

$$
\begin{aligned}
& F T(s)=\frac{1}{E C_{P}(s)} \cdot \sum_{e_{l} \in P} \frac{N E_{l}}{P A_{x y}^{l}} . \\
& N E_{l}=\left\{\begin{array}{ll}
1 & \text { Nashequilibrim } \\
>1 & \text { otherwise }
\end{array} .\right.
\end{aligned}
$$

Obviously, the smaller its fitness value, the better the solution.

The information transfer target set is defined as follows:

$$
T=\left\{s \mid(s \in I) \wedge\left(F T(s)=F T^{*}\right)\right\} .
$$

Here, $I$ is solution space, $F T *$ is the smallest fitness value of solutions in $I$.

Define the distance between two nodes as follows:

$$
d\left(s_{b}, s_{c}\right)=\left\|s_{b}-s_{c}\right\| .
$$

Here, $s_{b}, s_{c} \in S,\left\|s_{b}-s_{c}\right\|$ is the Hamming distance between $s_{b}$ and $s_{c}$.

Define the set of solutions in its $\ell$ neighborhood of $s_{b}$ as follows:

$$
\zeta^{\ell}\left(s_{b}\right)=\left\{s_{c} \mid\left(s_{c} \in S\right) \wedge\left(0<\left\|s_{b}-s_{c}\right\| \leq \ell\right)\right\} .
$$

Then, use $\overline{\zeta^{\ell}\left(s_{b}\right)}$ to represent the set of solutions not in its $\ell$ neighborhood of $s_{b}$.

\subsection{Local Short Conjunctive Search Operator}

When $\ell$ is small, this operator is used to transfer information from $s_{b}(j)$ to $s_{b}(j+1)$ which is the closest to $T$ in $\zeta^{\ell}\left(s_{b}(j)\right)$, denoted as $s_{b}(j+1) \leftarrow \Psi\left(s_{b}(j)\right)$. In this paper, 
take $N_{l e n}(j)<\left|\zeta^{\ell}\left(s_{b}\right)\right|$ nodes from $\zeta^{\ell}\left(s_{b}(j)\right)$ randomly to construct a temporary local transfer network for local search. It is described in algorithm 3.1.

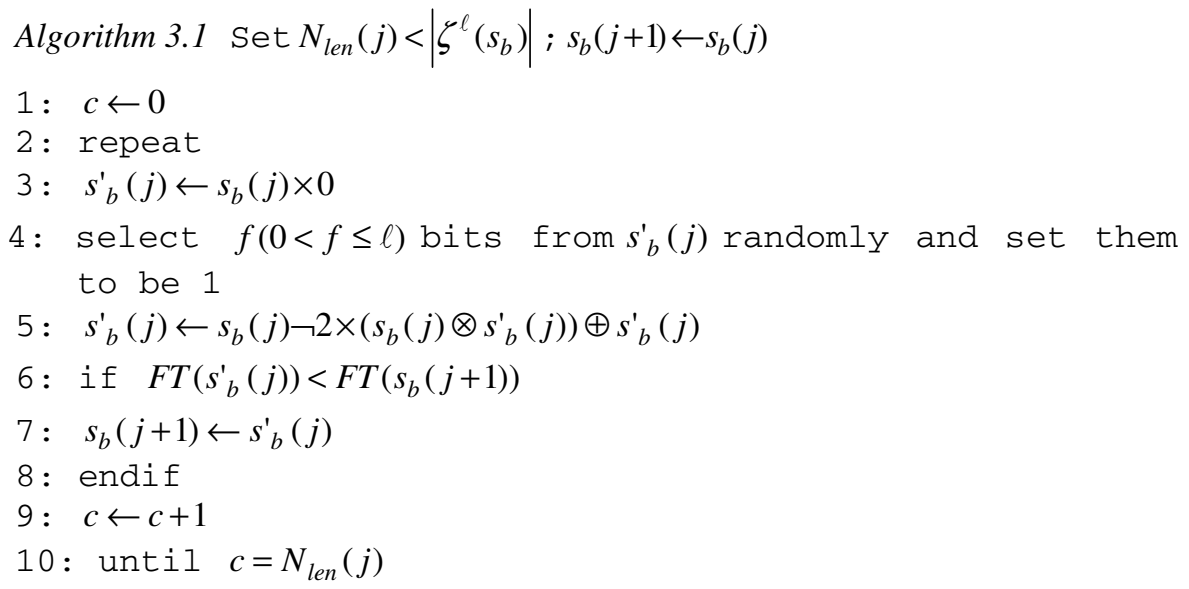

Here, $j$ is the iteration times, $s_{b}(j) \times 0$ is to obtain a temporary string with each bit to be set $0, \oplus$ represents "add bit by bit", ᄀ represents "subtract bit by bit", and $\otimes$ represents "multiply bit by bit".

\subsection{Random Long Conjunctive Searching Operator}

When $\ell$ is large, this operator is used to select a node $s_{b}^{\prime}(j)$ in $\overline{\zeta^{\ell}\left(s_{b}\right)}$ randomly by a preset probability as information transfer object node of $s_{b}(j)$, which is denoted as $S^{\prime}(j) \leftarrow \Gamma(S(j))$. It is described in algorithm 3.2.

Algorithm 3.2 set global long conjunctive probability $p_{l e n}$ and $\ell$

1: $b \leftarrow 0$

2: repeat

$3: s^{\prime}{ }_{b}(j) \leftarrow s_{b}(j)$

4: $\quad p \leftarrow \operatorname{rand}(0-1)$

5: if $p_{l e n}<p$ and $s_{b}(j)$ is not the optimum in the current node set

6:generate two integers $\mu$ and $v$ randomly, $1 \leq \mu<v \leq$ len , $|\mu-v|>\ell$

$7: \quad s_{b}(j) \leftarrow s_{b}^{\prime}(j) \mid \begin{aligned} & v \\ & \mu\end{aligned}$

8: endif

9: $b \leftarrow b+1$

10: until $b=N$ 
In the above, the forth sentence is to generate a random number which is evenly distributed between 0 and 1 , the seventh sentence is to reverse the bit order from the $\mu$ th to the $v$ th bit in $s_{b}^{\prime}(j)$.

\subsection{Algorithm Procedure}

The procedure of the proposed QoS unicast routing algorithm is described as follows:

Step1: Set the node set size to be $N$; set $p_{|E|}$ and $\ell$.

Step2: Generate the initial node set $S(0)=\left\{s_{1}(0), s_{2}(0), \cdots, s_{N}(0)\right\}$ randomly according to section 3.1, i.e., generate $N$ initial unicast paths.

Step3: For each node $s_{b}(0) \in S(0)$, compute the user utility and the network provider utility on each edge according to formula (23) and (25) in [21], and play game according to section 2.4 in [21].

Step4: Compute $F T\left(s_{b}(0)\right)$ for each node $s_{b}(0) \in S(0)$.

Step5: Judge whether $s_{b}(0)$ meets the constraints defined by formula (36)-(39) in [21] or not: if not so, regenerate it and go to Step4.

Step6: Record the best fitness value $F T^{b}$ of the nodes in the current node set.

Step7: Set the iteration times to be $I t r$, set $\mathcal{E}, j=0$.

Step8: If $j \leq \operatorname{Itr}$ and $\left|F T^{*}-F T^{b}\right| \leq \varepsilon$ (termination criteria)is not met, go to Step9, otherwise go to Step17.

Step9: Construct the temporary node set $S^{\prime}(j), S^{\prime}(j) \leftarrow S(j)$.

Step10: execute the algorithm 3.2, $S^{\prime}(j) \leftarrow \Gamma\left(S^{\prime}(j)\right)$.

Step11: $b=0$

Step12: execute the algorithm 3.1 to $s_{b}(j), \quad s_{b}{ }_{b}(j+1) \leftarrow \Psi\left(s_{b}{ }_{b}(j)\right)$, $s_{b}^{\prime}(j) \in S^{\prime}(j)$.

Step13: If $F T\left(s_{b}^{\prime}(j+1)\right)<F T\left(s_{b}(j)\right) \quad, \quad s_{b}(j+1) \leftarrow s_{b}^{\prime}(j+1) \quad, \quad$ otherwise $s_{b}(j+1) \leftarrow s_{b}(j)$.

Step14: $b=b+1$. If $b<N$, go to Step12, otherwise go to Step15.

Step15: Update $F T^{b}$ with the best fitness value of the nodes in the current node set.

Step16: $j=j+1$, go to Step8.

Step17: Output the optimal node as the problem solution, the algorithm ends.

Specially, when $0<\left|F T^{*}\right|<1$, termination criteria changes to $\left|F T^{*}-F T^{b}\right|<\varepsilon\left|F T^{*}\right|$.

\section{Simulation Research}

Simulations of the proposed QoS unicast routing scheme have been done on NS2 (Network Simulator 2)[23] and simulation results have shown that it has better 
performance when its main parameters are set to be the following values[22]: $\varepsilon=0.0005, k=2, \sigma_{1}=3, \sigma_{2}=3, \alpha_{B}=1 / 3, \alpha_{D}=1 / 3, \alpha_{L}=1 / 3, n=6, m=4, \delta=0.8$, $k_{1}=0.6, k_{2}=0.4, \lambda_{1}=0.6, \lambda_{2}=0.6, \rho=0.5, \alpha=0.5, \beta=0.5$.

Assume that there are three network providers providing satellite, cellular and fixed links respectively, that is, there are three kinds of links for a user to choose on each hop along the path. The proposed scheme, the proposed microeconomics based fuzzy unicast QoS routing scheme in [24], and the unicast routing scheme based on Dijkstra algorithm [8] have been simulated on some physical and virtual network topologies, called A, G and D schemes below for short. Compared with A scheme, G scheme takes imprecise network status and both-win of the network provider utility and the user utility into account but does not consider imprecise user QoS requirement and $\mathrm{ABC}$. Simulation results have shown that A scheme has the better performance. Comparison results on QoS unicast routing request succeeded rate(RSR), user utility(UU), satellite link provider utility(SPU), cellular link provider utility(CPU), fixed link provider utility(FPU), network provider utility(NU=SPU+CPU+FPU), comprehensive utility $(\mathrm{CU}=\mathrm{UU}+\mathrm{NU})$, Pareto optimum ratio under Nash equilibrium(PRN) achieved by $\mathrm{A}, \mathrm{G}$ and $\mathrm{D}$ schemes over CERNET topology(T1), CERNET2 topology(T2), GÉANT topology(T3) and one virtual topology(T4, generated by Waxman2[25] with average node degree 3.5) are shown in Table 1. From Table 1, it can be concluded that the proposed scheme has the better performance, especially when topologies are complex.

Table 1(1). Comparison Results

\begin{tabular}{l|l|l}
\hline \multirow{2}{*}{ Metrics Topology } & T1 & T2 \\
\cline { 2 - 3 } & A:G:D & A:G:D \\
\hline RSR & $1.1624: 1.1136: 1.0000$ & $1.1427: 1.0848: 1.0000$ \\
UU & $1.1327: 1.0816: 1.0000$ & $1.1029: 1.0937: 1.0000$ \\
SPU & $1.0657: 1.0411: 1.0000$ & $1.0531: 1.0384: 1.0000$ \\
CPU & $1.1576: 1.1295: 1.0000$ & $1.1465: 1.1152: 1.0000$ \\
FPU & $1.1253: 1.0773: 1.0000$ & $1.1187: 1.0692: 1.0000$ \\
NU & $1.1369: 1.0873: 1.0000$ & $1.1057: 1.0632: 1.0000$ \\
CU & $1.1347: 1.0866: 1.0000$ & $1.1036: 1.0793: 1.0000$ \\
PRN & $4.4091: 3.6819: 1.0000$ & $4.3519: 3.7262: 1.0000$ \\
\hline
\end{tabular}

Table 1(2). Comparison Results

\begin{tabular}{l|l|l}
\hline \multirow{2}{*}{ Tetrics } & T3 & T4 \\
\cline { 2 - 3 } A:G:D & A:G:D \\
\hline RSR & $1.1935: 1.1218: 1.0000$ & $1.2129: 1.1694: 1.0000$ \\
UU & $1.1737: 1.1033: 1.0000$ & $1.1859: 1.1097: 1.0000$ \\
SPU & $1.0814: 1.0548: 1.0000$ & $1.0894: 1.0627: 1.0000$ \\
CPU & $1.1859: 1.1348: 1.0000$ & $1.2065: 1.1674: 1.0000$ \\
FPU & $1.1468: 1.0961: 1.0000$ & $1.1722: 1.1283: 1.0000$ \\
NU & $1.1562: 1.1409: 1.0000$ & $1.1757: 1.1376: 1.0000$ \\
CU & $1.1631: 1.1274: 1.0000$ & $1.1991: 1.1184: 1.0000$ \\
PRN & $4.8439: 3.9644: 1.0000$ & $5.2161: 4.2493: 1.0000$ \\
\hline
\end{tabular}




\section{Conclusion}

In this paper, by introducing knowledge of fuzzy mathematics, probability theory and gaming theory, a QoS unicast routing scheme with ABC supported is proposed based on small-world optimization algorithm. Under imprecise network status and inaccurate user QoS requirement, it tries to search for a QoS unicast path to make both the network provider utility and the user utility achieve or approach Pareto optimum under Nash equilibrium. Simulation results have shown that the proposed scheme is both feasible and effective with better performance. In future, our study will focus on improving its practicality, developing its prototype system and extend it to multicast scenario.

\section{References}

1. Daoud, F., Mohan, S.: Challenges of Personal Environments Mobility in Heterogeneous Networks. Mobile Networks and Applications 8(1), 7-9 (2003)

2. Lu, W.W.: Open Wireless Architecture and Enhanced Performance. IEEE Communications Magazine 41(6), 106-107 (2003)

3. Gustafsson, E., Jonsson, A.: Always Best Connected. IEEE Wireless Communications 10(1), 49-55 (2003)

4. Fodor, G., Eriksson, A.: Aimo Tuoriniemi: Providing Quality of Service in Always Best Connected Networks. IEEE Communications Magazine 41(7), 154-163 (2003)

5. Zahariadis, T.B., Vaxevankis, K.G., Tsantilas, C.P., et al.: Global Roaming in NextGeneration Networks. IEEE Communications Magazine 40(2), 145-151 (2002)

6. Quan, X.T., Zhang, J.: Theory of Economics Game. China Machine Press, Beijing (2003)

7. Wang, Z., Crowcroft, J.: Quality of Service Routing for Supporting Multimedia Applications. IEEE Journal on Selected Areas in Communications 14(7), 1288-1294 (1996)

8. Wang, Z., Crowcroft, J.: QoS Routing for Supporting Resource Reservation. IEEE Journal on Selected Areas in Communications 14(7), 1228-1234 (1996)

9. Dijkstra, E.W.: A Note on Two Problems in Connection with Graphs. Numerical Mathematics 1 (1959)

10. Salama, H.F., Reeves, D.S., Viniotis, Y.: A Distributed Algorithm For Delay-Constrained Unicast Routing. IEEE/ACM Transactions on Networking 8(2), 239-250 (2000)

11. Kim, M., Bang, Y.-C., Choo, H.: New Parameter for Balancing Two Independent Measures in Routing Path. In: Laganà, A., Gavrilova, M., Kumar, V., Mun, Y., Tan, C.J.K., Gervasi, O. (eds.) ICCSA 2004. LNCS, vol. 3046, Springer, Heidelberg (2004)

12. Gelenbe, E.: An Approach to Quality of Service. In: Aykanat, C., Dayar, T., Körpeoğlu, İ. (eds.) ISCIS 2004. LNCS, vol. 3280, Springer, Heidelberg (2004)

13. Orda, A., Sprintson, A.: QoS Routing: the Precomputation perspective, pp. 128-136. IEEE Computer Society Press, Los Alamitos (2000)

14. Cormen, T.H., Leiserson, C.E., Rivest, R.L.: Introduction to Algorithms. MIT Press, Cambridge, MA (1990)

15. Shin, K.G., Chou, C.C.: A Distributed Route-Selection Scheme for Establishing RealTime Channels. In: International Conference on High Performance Networking, pp. 319 329 (1995)

16. Chen, S., Nahrsted, K.: On Finding Multi-Constrained Paths. In: Proceedings of IEEE ICC'98, pp. 874-899 (1998) 
17. Wang, X.W., Wang, Z.J., Huang, M., et al.: Quality of Service Based Initial Route Setup Algorithms for Multimedia Communication. Chinese Journal of Computers 24(8), 830 837 (2001)

18. Barolli, L., Koyama, A. (eds.): A Genetic Algorithm Based Routing Method Using Two QoS Parameters, vol. 8(1), pp. 7-11. IEEE Computer Society, Los Alamitos (2002)

19. Dorigo, M.: Ant Algorithms Solve Difficult Optimization Problems. In: Advances in Artificial Life: 6th European Conference, pp. 11-22. Czech Republic, Prague (2001)

20. Cui, Y., Wu, J.P.: A QoS Routing Algorithm by Applying Simulated Annealing. Journal of Software 14(5), 877-884 (2003)

21. Wang, X.W., Gao, N., Cai, S.X., Huang, M.: An Artificial Fish Swarm Algorithm Based and ABC Supported QoS Unicast Routing Scheme in NGI. In: Min, G., Di Martino, B., Yang, L.T., Guo, M., Ruenger, G. (eds.) Frontiers of High Performance Computing and Networking - ISPA 2006 Workshops. LNCS, vol. 4331, pp. 205-214. Springer, Heidelberg (2006)

22. Li, X.L., S, Z.J, Q, J.X.: An Optimizing Method Based on Autonomous Animals: Fishswarm Algorithm. Systems Engineering-Theory \& Practice 22(11), 32-38 (2002)

23. Xu, L.M., Pang, B., Zhao, R.: NS and Network Simulation, pp. 1-9. Posts \& Telecom Press, Beijing (2003)

24. Wang, X.W., Hou, M.J., Wang, J.W., et al.: A Microeconomics-based Fuzzy QoS Unicast Routing Scheme in NGI. In: Yang, L.T., Amamiya, M., Liu, Z., Guo, M., Rammig, F.J. (eds.) EUC 2005. LNCS, vol. 3824, pp. 1055-1064. Springer, Heidelberg (2005)

25. Waxman, B.M.: Routing of Multipoint Connections. IEEE Journal on Selected Areas in Communications 6(11), 478-489 (1988) 